\title{
Uso de las comunidades de marca online para la comunicación con públicos del sector de automoción
}

Use of the online

brand communities

for the communication

with the public of the automotive sector

Rafael Anaya Sánchez Universidad de Málaga rafael.anaya@uma.es

Rocío Aguilar Illescas Universidad de Málaga rocio.aguilar@uma.es

Sebastián Molinillo Jiménez Universidad de Málaga

smolinillo@uma.es
Sánchez, R., Aguilar, R. y Molinillo, S. (2014). Uso de las comunidades de marca online para la comunicación con públicos del sector de automoción. Revista Internacional de Investigación en Comunicación aDResearch ESIC. No $10 \mathrm{Vol} 10$. Segundo semestre, julio-diciembre 2014. Págs. 110 a 125

DOI: 10.7263/ADRESIC.010.006 
RESUMEN

\section{Clasificación JEL: M31 Marketing, M37 Advertising Palabras clave:}

Comunidades virtuales de marca, marketing online, redes sociales, empresas de automoción
El análisis de las comunidades virtuales de marca (CVM) está en un continuo crecimiento, gracias a su capacidad de fortalecer las relaciones con los distintos públicos de la empresa y de mejorar la imagen de marca. Son muchos los estudios que se han centrado en el análisis de sus ventajas para las organizaciones, fundamentalmente como herramienta de comunicación y de soporte a otras acciones comerciales. Hasta el momento se han analizado comunidades de productos de muy diversa naturaleza, desde marcas de alta preferencia (p. ej.: Apple), hasta productos de conveniencia (p. ej:: Nutella). Las marcas de automoción han sido el centro de parte de estos estudios (Harley-Davidson, Mini, Yamaha, etc.) poniendo de manifiesto el interés de las empresas de este importante sector económico, por reforzar la lealtad a la marca. No obstante, hasta el momento no ha sido estudiado el uso conjunto de las comunidades virtuales por las empresas de un sector sino que se han analizado casos independientes. Para responder a este vacío en el conocimiento de las comunidades virtuales de marca en el sector de automoción, el presente artículo analiza los usos que de ellas hacen 19 marcas de automóviles y de motocicletas, como medios de comunicación. La investigación concluye que las CVM se están utilizando principalmente para la comunicación de eventos, acciones y promociones, tal vez excesivamente, sin que se visualice claramente el fortalecimiento de la imagen, la interactividad ni la identificación de los usuarios con la comunidad, por lo que no se aprovecha convenientemente el potencial de este canal de comunicación. Se sugieren algunos cambios para mejorar el uso de las CVM.

\section{ABSTRACT}

\section{Jel Classification: \\ M31 Marketing \\ M37 Advertising Key words:}

Virtual Brand

Communities,

online marketing,

social networks,

automotive companies
The analysis of the Virtual Brand Communities (VBC) is continuously growing due to their ability to support the relationships with the different business public and to improve the brand image. There are numerous studies focused on the analysis of their advantages for the organizations, mainly as a communication tool and support for other commercial actions. Up to now product communities of very diverse nature, from high-preference products (e.g. Apple) to convenience products (e.g. Nutella) have been studied. Automotive have also been the subject of study of some of these analyses (Harley-Davidson, Mini, Yamaha, etc.) showing the interest of companies from this economic sector in strengthening brand loyalty. However, the joint use of the virtual communities by companies of this sector has not been studied yet as only particular cases have been analysed.

In order to respond to the lack of knowledge of Virtual Brand Communities in the automotive sector, this article analyses the uses that 19 car and motorcycle make brands make of them as means of communication. The research concludes that VBC are mainly being used to announce events, actions and promotions, maybe in excess, without clearly visualizing the strengthening of the brand image, the interactivity or the users' identification with the community, so the potential of this means of communication is not fully utilized. Some changes are suggested in order to improve the use of VBC. 


\section{Introducción}

La evolución de Internet ha hecho que la web pase de ser un lugar para la difusión unidireccional de información, a un espacio de diálogo y comunicación. En este contexto, en los últimos tiempos las comunidades virtuales (CV) se han convertido en una de las principales herramientas de comunicación online. No obstante, su origen en los primeros días de la Red era muy distinto ya que las compañías de software desarrollaron estos espacios para crear grupos de usuarios que les permitieran testar los programas (Weber, 2007). Por lo tanto, las CV comenzaron como eventos espontáneos, no comerciales y sociales (Rheingold, 1993), para compartir experiencias, opiniones y conocimientos (Bickart y Schindler, 2001). Sin embargo, su expansión y penetración como instrumento de comunicación ha despertado el interés de varias disciplinas científicas como la sociología, la psicología, la economía o el marketing (Li, 2004; Berger y Messerschmidt, 2009), conformándose como uno de los canales con mayor crecimiento en los últimos años (Zaglia, 2013).

Rheingold (1993) definió las CV como agregaciones sociales que surgen de la red cuando un número suficiente de personas realiza discusiones públicas, durante un periodo duradero y con suficientes sentimientos entre sí para formar sitios Web de relaciones personales en el ciberespacio. Preece (2000), Lee, Vogel y Moez (2003) y Porter (2004) incluyeron en las CV la participación de las empresas que perseguían compartir información y comunicarse con otras empresas y usuarios.

Las CV han crecido exponencialmente como resultado del mayor uso de herramientas de contacto entre consumidores para conseguir información y comunicarse (Casaló, Flavián y Guina- líu, 2007), lo que hace que las empresas traten de entrar en el diálogo generado en la Web, considerando que cuanto más fuerte sea el diálogo y más usuarios hablen de ellas, más fuerte será la marca (Weber, 2007). Por lo tanto, la componente social de las CV puede desarrollarse alrededor de una marca (Sicilia y Palazón, 2010), ayudando al consumidor a conformar y expresar su personalidad y a articular valores compartidos con otras personas (Wiegandt, 2009). De este modo, los consumidores utilizan las comunidades para tomar decisiones sobre productos y marcas (Kozinets, 2002), lo que ha llevado a las empresas a crear comunidades virtuales de marca (CVM) para apoyar la estrategia de marketing (Powell, Groves y Dimos, 2011).

En este contexto el objetivo de este trabajo es conocer la presencia de las empresas del sector de automoción en las CVM a través de las redes sociales y los usos que de ellas se suelen hacer en el ámbito de la de comunicación.

\section{Aproximación al concepto de comunidades virtuales de marca (CVM)}

Las CVM han servido a los consumidores para compartir experiencias, intercambiar información, solucionar problemas y conocer a consumidores similares (McWilliam, 2000). Las comunidades de marca en Internet se establecieron en la Web 1.0 en portales gestionados por empresas o iniciados por consumidores independientes a la empresa (Jang et al., 2008). A partir del crecimiento de la Web Social muchas compañías y usuarios empiezan a usar los sitios web como medios para crear y organizar comunidades en torno a las marcas (Kaplan y Haenlein, 2010; Laroche et al., 2012). Así, una comunidad nace basada en su activo básico: la marca, y pos- 
teriormente crece construyendo relaciones entre los miembros (Jang et al., 2008).

Muñiz y O'Guinn (2001) definen las CVM como una comunidad especializada y no vinculada a un lugar geográfico, basada en una estructura de relaciones sociales entre admiradores de una marca, de manera que las relaciones se producirían entre las marcas y los consumidores, pero también entre los propios consumidores. Por otra parte, McAlexander, Schouten y Koenig (2002) extienden las relaciones que se establecen entre el consumidor y la empresa incluyendo la interacción entre el consumidor y el propio producto. De este modo, las CVM se convierten en herramientas básicas para la creación de relaciones entre consumidores y marcas (Bagozzi y Dholakia, 2002; Dholakia, Bagozzi y Pearo, 2004) permitiendo: aumentar la lealtad y aprender de los consumidores (Laroche et al., 2012); maximizar las oportunidades de atraer y colaborar con los consumidores (Franke y Shah, 2003); influir en las acciones y evaluaciones de los miembros (Muñiz y Schau, 2005); y diseminar la información (Brown, Kozinets y Sherry, 2003). Así, las CVM son reconocidas por las empresas como un medio eficiente de comunicación con consumidores, obteniendo ideas y mejorando las relaciones con proveedores (Jang et al., 2008). Además permiten instaurar nuevos canales de comunicación con los distintos públicos y crear nuevas formas de vinculación con los usuarios (Andersen, 2005).

Existen diferentes tipos de CVM aunque se suelen dividir en dos grupos según sean promovidas por consumidores o por empresas (Muñiz y O'Guinn, 2001; Henri y Pudelko, 2003; Leimeister, Sidiras y Krcmar, 2004; Porter, 2004; Sung et al., 2010). Las comunidades iniciadas y administradas por los usuarios nacen por la vo- luntad y trabajo de estos (Shang, Chen y Liao, 2006; Jang et al., 2008), debido a que suelen ser consumidores entusiastas de la marca que quieren compartir información sobre sus productos y establecer relaciones con otros consumidores similares a ellos (Shang, Chen y Liao, 2006). Por su parte, las comunidades creadas directamente por las empresas tienen por objetivo establecer relaciones con consumidores e inducirles a generar un feedback útil para la empresa (McWilliam, 2000; Jang et al., 2008; Sung et al., 2010). En ellas se suelen ofrecer promociones, cupones o permitir la participación en eventos, entre otras acciones, con el fin de comunicarse activamente con la comunidad.

En cuanto a la plataforma empleada, la mayoría de las CVM están alojadas en redes sociales ya que, además de ofrecer un soporte adecuado, son ampliamente usadas por los consumidores (Zaglia, 2013).

\section{La motivación de los participantes en las CVM}

El crecimiento del uso de las CVM se debe en gran parte al crecimiento de Internet y de los medios sociales (Smedberg, 2008), pero es muy importante conocer cuáles son realmente las motivaciones que llevan a una persona a hacerse miembro de ellas, y una vez dentro, qué le hace interactuar y mantener su pertenencia (SánchezFranco y Rondán-Cataluña, 2010). Este interés, desde la perspectiva de la marca, radica en que su comprensión ayudaría a aumentar la lealtad del consumidor (Dick y Basu, 1994) y a lograr así una mayor estabilidad de la empresa (Keating, Rugimbana y Quazi, 2003).

Según Bagozzi y Dholakia (2006) las personas tienen diferentes razones para entrar a formar parte y participar en las comunidades pero, 
en opinión de Zheng, Zhao y Stylianou (2013), todas relacionadas con el logro de la satisfacción de sus necesidades. Así, los investigadores suelen coincidir en que las personas y organizaciones participan en comunidades virtuales básicamente para interactuar y comunicarse entre sí (Smedberg, 2008) debido a que tienen intereses, un propósito o una experiencia común (Preece, 2000; Berger y Messerschmidt, 2009), de manera que alcanzan nuevas relaciones sociales (Wellman et al., 1996; Jung y Kang, 2010; Wang y Chen, 2012).

Por otra parte, son muchos los factores argumentados como elementos básicos en la captación y mantenimiento de usuarios. Etzioni (1996) y Casaló, Flavián y Guinalíu (2010) indican la identificación del propio individuo con el grupo, mientras que Farquhar y Rowley (2006) destacan que es necesaria la existencia de una serie de recursos y un firme compromiso por parte de los miembros de la comunidad. En cualquier caso, la satisfacción se perfila como un resultado esencial de la integración (Casaló, Flavián y Guinalíu, 2011) y además tiene un efecto directo como antecedente sobre la lealtad a la CV (Henning-Thurau, Winner y Gremmler, 2002; Yen y Gwinner, 2003). No obstante, el uso de una determinada comunidad estará influenciado por el entretenimiento que ésta aporte al usuario (Cheung, Chiu y Lee, 2011; Lin y Lu, 2011). Por ello, las experiencias entretenidas en una comunidad virtual tendrán un impacto positivo en la generación de relaciones de confianza entre los participantes de la comunidad (Yang, 2010). De tal forma, el entretenimiento puede atraer al consumidor a utilizar, generar o hacer aportaciones a los contenidos online que tengan relación con la marca (Muntinga, Moorman y
Smit, 2011) y, además, puede incentivarlo a regresar al sitio web (Raney et al., 2003).

\section{Las CVM como canal de comuni- cación}

En la última década los canales de comunicación de las empresas han cambiado profundamente debido a la expansión de la Web Social. Uno de los elementos más afectadosha sido la publicidad tradicional ya que ha perdido credibilidad, impacto e interés por parte de las empresas que pagan por ella (Clemons, 2009). Esto se debe a que las compañías ya no tienen el control de lo que los consumidores leen, escuchan o hablan, además de que los consumidores tratan de evitar la publicidad por considerarla masiva y poco segmentada (Weber, 2007).

En Internet las 4Ps tienen un comportamiento diferente al mundo offline (Kalyanam y McIntyre, 2002). Sin embargo, con frecuencia la promoción o comunicación online se ha convertido en una amplificación natural de la promoción offline, aunque los consumidores están logrando cada vez un mayor control sobre los medios de comunicación en sus decisiones de compra (Kucuk y Krishnamurthy, 2007). Por ello, si las empresas quieren adoptar la nueva concepción de la comunicación, tendrán que realizar una serie de cambios en su estructura para adaptarse a la versión 2.0 (Cortés y Martínez-Priego, 2010). De este modo, se deberá pasar de la publicidad a la conversación, por lo que la empresa tiene que utilizar nuevas formas de transmitir el mensaje hasta el cliente en condiciones de igualdad, acudiendo a los lugares donde éste se encuentra en Internet. En consecuencia, la empresa buscará al individuo en las comunidades, ya que la nueva tecnología social consigue que los usuarios puedan interactuar en grandes grupos, pudiendo 
compartir, hablar y generar opiniones entre ellos (Cortés y Martínez-Priego, 2010).

Por lo tanto, la Web Social se está postulando como centro de la actividad de comunicación de las empresas convirtiendo a los usuarios en medios de comunicación a medida que producen contenidos (Weber, 2007). Esto provoca que las organizaciones difundan sus mensajes directamente a través de la web sin confiar exclusivamente en la publicidad tradicional (Meerman, 2007). Esta nueva situación posibilita que existan múltiples partes publicando mensajes relacionados con la marca, lo que acarrea que la empresa ya no posea el control completo del mensaje (Powell, Groves y Dimos, 2011), de manera que se crea un nuevo canal de comunicación en el que los clientes desempeñan un papel colaborador fundamental (Hoffman y Novak, 1997) y las empresas dejan de ser meras proveedoras de información (Meerman, 2007).

El aumento de la importancia de los medios sociales como fuentes de información ha llevado a los anunciantes y a los propietarios de los medios de comunicación tradicionales, a buscar formas alternativas de llegar a los consumidores de forma más persuasiva (Clemons, 2009). Lo anterior no siempre se consigue, ya que en muchos casos su uso se limita a reproducir los formatos digitales que aparecen en sitios web estáticos, sin adaptarlos a las CV. Además, la publicidad en los medios sociales puede ser percibida de forma negativa, ya que los usuarios se encuentran en lugares en los que la publicidad puede ser percibida como una invasión (De Salas, 2009). Esto es debido a que el usuario de redes sociales virtuales se sitúan en un estado mental proclive a las relaciones y a la socialización, pero no a la compra (Dans, 2007). Así, para que la publicidad en redes sociales virtuales sea aceptada por el usuario debe entretener, divertir, seducir, ser grata y oportuna, fomentando la viralidad, ya que en caso contrario el usuario la rechazará (De Salas, 2009).

En este sentido, para lograr la participación de los usuarios y su positiva reacción ante acciones promocionales en las CVM se deberán considerar dos aspectos fundamentales:

- Evitar la percepción de entorno controlado por el sponsor de la comunidad. Los consumidores participan en las comunidades de marca debido a que confían en mayor medida en otros consumidores que en las empresas propietarias de las marcas (Kotler, Kartajaya y Setiawan, 2010). Ante esto, las empresas deben ajustar sus modelos de negocio para aprovechar el potencial disponible (Hanna, Rhom y Crittenden, 2011) y participar en las conversaciones (Muñiz y O'Guinn, 2001; Weber, 2007). La organización querrá controlar lo que se dice sobre ella en sus comunidades (Singh y Sonnenburg, 2012), pero deberá dejar cierta libertad a sus usuarios para expresarse, ya que la forma en que se haga el control influirá en la credibilidad que los consumidores darán a la comunidad (Wirtz et al., 2013).

- No abusar del uso comercial de la comunidad. La realización de actividades comerciales en las comunidades puede suponer una intromisión (Clemons, 2009), aunque cuando un consumidor entra en una comunidad organizada por una marca estará dispuesto a establecer relaciones, otorgando un feedback útil a la empresa (McWilliam, 2000; Jang et al., 2008; Sung et al., 2010) y permitiendo un posible canal de promoción de productos y servicios (Shang, 
Chen y Liao, 2006). La organización a la hora de gestionar su participación en comunidades deberá comprender los motivos de participación de sus usuarios y adaptar sus planes de marketing, (Kozinets, 2002; Koh y Kim, 2004; MartínezLópez y Luna, 2008; Powell, Groves y Dimos, 2011), sin que el usuario perciba que el sponsor se aprovecha de la comunidad (Porter y Donthu, 2008).

\section{Metodología}

En este trabajo se analizará el uso de las CVM como canal de comunicación a través del método de estudio de casos, dado que su validez ha sido contrastada en investigaciones previas como la desarrollada por Flavián y Guinalíu (2005). El objetivo de este estudio es examinar cómo las marcas incrementan su capacidad de comunicación a través de las CVM, excluyendo otras acciones de comunicación que realizan en las redes sociales pero fuera de su CVM (por ejemplo: banners en Facebook o spots en Youtube). Específicamente, se analizará cómo se comunica, qué tipo de formatos se utilizan, cuál es el grado de adaptación de la comunicación a las particularidades del canal y qué interactividad hay con y entre los usuarios.

Con tal fin se estudiará el caso de las marcas de automoción, ya que en este sector se produce una importante vinculación entre el cliente y la marca (Marzocchi, Morandin y Bergami, 2012). Hasta el momento en el sector se han investigado algunas comunidades como, por ejemplo: Yamaha Rl (Félix, 2012), Volswagen (Brown, Kozinets y Sherry, 2003), Harley-Davidson (Schouten y McAlexander, 1995; Bagozzi y Dholakia, 2006), Saab y Jeep (Muñiz y O'Guinn, 2001), Ducati (Marzocchi, Morandin y Bergami,
2012) o MINI Cooper (Yeh y Choi, 2011). Sin embargo, por el momento no se ha realizado ninguna investigación que analice comparativamente el uso que las marcas del sector hacen de las comunidades virtuales. Por lo tanto, para responder a ese vacío en el conocimiento de las CVM en este trabajo se analizará un amplio conjunto de 19 CVM del sector de automoción, específicamente vehículos y motocicletas, que han sido creadas y son gestionadas por las propias marcas en español en España (véase tabla 1). Las marcas de turismos seleccionadas se encuentran entre las 20 marcas más vendidas a nivel nacional (MSI, 2013). Así, se han incluido las ocho marcas con mayores cifras de ventas en el sector, y las tres primeras marcas en el segmento de berlinas y alta gama (Mercedes, Audi y BMW) por considerar que al ser el perfil del cliente diferente, también lo podrían ser las estrategias de comunicación de las empresas. En cuanto a las marcas de motocicletas elegidas para la realización de este estudio, todas aparecen entre las 15 más vendidas en España (Anesdor, 2014).

En cuanto a las plataformas se han considerado solo las CVM presentes en cinco redes sociales: Facebook, Google+, Instagram, Twitter y Youtube. El motivo de elección de dichas redes se basa en que son las de mayor notoriedad (IabSpain, 2014) y entre ellas se excluye Tuenti porque gran parte de sus usuarios son jóvenes menores de 18 años (Apaolaza et al., 2013; Marta, Martínez y Sánchez, 2013) y, por lo tanto, la plataforma es menos atractiva para las marcas de automoción. Por otra parte, se han considerado las CVM que representan la actividad de las marcas en España, no las generales de la compañía a nivel mundial ni las gestionadas directamente por los usuarios sin la participación corporativa de la marca. 


\section{Estudio de casos}

De las 19 empresas incluidas en el estudio, solo 5 disponen de comunidades propias gestionadas por la empresa en español, en todas las redes sociales analizadas: Citroën, Mercedes, Seat, Toyota, Volkswagen (esta última cuenta con Facebook propio para el modelo New Beetle). En general, el uso de las redes sociales presenta un elevado número de parti- cularidades en el conjunto de marcas analizadas. Así, por ejemplo, Renault en Google+ tiene comunidades de modelos pero no una general y Honda dispone de comunidades separadas para coches y motos. Además, aparte de las plataformas de las redes sociales virtuales, algunas de las empresas estudiadas han generado comunidades a partir de páginas webs o blogs.

\section{Tabla 1 • CVM de automoción en las principales redes sociales}

\begin{tabular}{|c|c|c|c|c|c|c|}
\hline Marca & Facebook & Google+ & Instagram & Twitter & Youtube & Web/blog \\
\hline Aprilia & 0 & & 0 & 0 & 0 & \\
\hline Audi & ○ & ○ & 0 & 0 & - & \\
\hline BMW & - & & 0 & - & - & \\
\hline Citroën & 0 & 0 & ○ & - & 0 & \\
\hline Ford & 0 & & 0 & - & ○ & 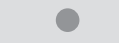 \\
\hline Harley-Davidson & 0 & & 0 & 0 & ○ & 0 \\
\hline Honda & 0 & 0 & 0 & - & ○ & \\
\hline Kawasaki & ○ & & 0 & 0 & 0 & \\
\hline KTM & 0 & 0 & & 0 & O & \\
\hline Mercedes-Benz & 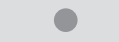 & 0 & 0 & 0 & 0 & \\
\hline Opel & 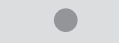 & 0 & 0 & - & 0 & - \\
\hline Peugeot & 0 & & 0 & 0 & 0 & - \\
\hline Piaggio & 0 & & 0 & & & \\
\hline Renault & 0 & 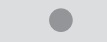 & & & 0 & ○ \\
\hline Seat & 0 & 0 & 0 & ○ & ○ & \\
\hline Suzuki & - & & 0 & & - & ○ \\
\hline Toyota & 0 & 0 & 0 & 0 & 0 & \\
\hline Volkswagen & 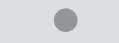 & 0 & - & 0 & ○ & ○ \\
\hline Yamaha & 0 & & & & ○ & \\
\hline = en español & \multicolumn{6}{|c|}{$\mathrm{O}=$ en inglés } \\
\hline
\end{tabular}


En cualquier caso, los objetivos comunicacionales de las CVM son muy diversos y, en cierta medida, coincidentes entre las diferentes plataformas. No obstante, el uso que se hace de la CVM varia atendiendo a sus propias características. Por lo tanto, a continuación se describen los usos más frecuentes de cada una de las plataformas consideradas (véase tabla 2).

\section{Facebook y Google+}

Aunque Facebook es la red con mayor penetración entre las empresas analizadas, dado que todas gestionan una comunidad en ella, aque-
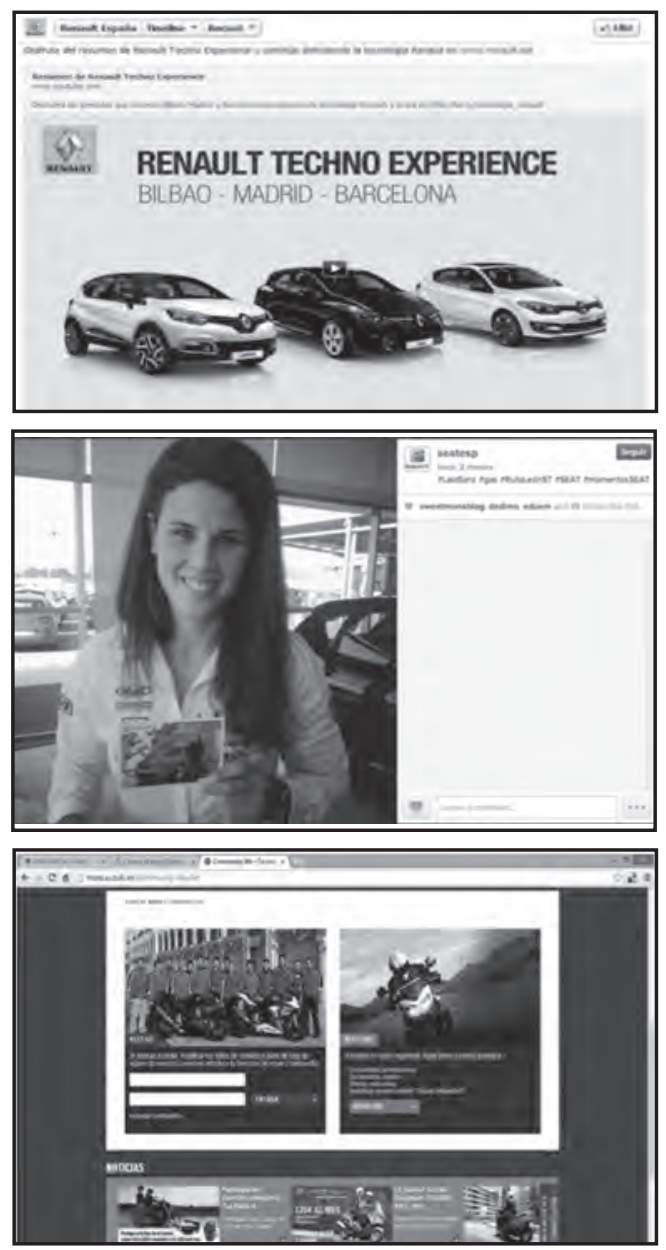

llas que también tienen presencia en Google+ reproducen los contenidos de la primera. En general su uso es corporativo, informando sobre nuevos productos, servicios, eventos, concursos, etc., pero también se emplea como medio en el que insertan formatos publicitarios. De esta forma, spots, anuncios comerciales gráficos, etc. son utilizados tradicionalmente, mientras que otros recursos como fotografías, enlaces o entradas en el muro de la comunidad, se utilizan más sutilmente.

\section{Instagram}

Esta plataforma se utiliza sobre todo para publicar imágenes y como lugar de creación de contenidos útiles para otras redes (p. ej.: twittear una imagen subida a Instagram). Estos contenidos suelen ser imágenes de vehículos con un diseño similar a la publicidad en Internet y en prensa escrita, aunque sin incluir un mensaje.
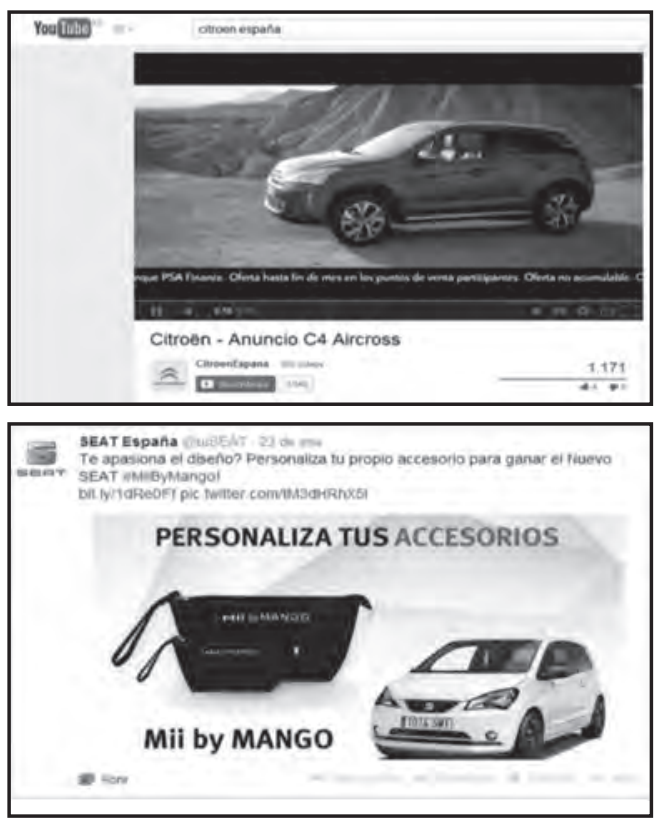


\section{Youtube}

Los contenidos compartidos en las comunidades basadas en Youtube se centran, en general, en las presentaciones de nuevos modelos, ya sea mediante vídeos de ferias, spots de televisión u otros generados específicamente para la red. En ellos el usuario participa mediante los comentarios del vídeo, votando o compartiendo, aunque hay que decir que no todas las empresas permiten comentarios sobre los vídeos.

\section{Twitter}

En esta red el comportamiento es más interactivo con los usuarios, aunque no todas las empresas gestionan esta relación por igual. Se observan notables diferencias en la frecuencia de la respuesta de menciones de seguidores, el uso de hashtags propios o el retwitteo de menciones o tweets de otros usuarios, llegando a tener más contenidos generados por usuarios que por la propia empresa. En general es ampliamente utilizada para informar sobre concursos, juegos $u$ otras actividades realizadas tanto online como offline por la empresa.

\section{Webs y blogs}

Como se ha comentado, además de las plataformas que ofrecen las redes sociales virtuales para crear y gestionar una comunidad virtual, algunas de las empresas estudiadas han generado comunidades, principalmente a partir de páginas webs. Estas comunidades suelen utilizarse para dudas y preguntas, publicidad, difusión de revistas e información diversa.

\section{Tabla 2 - Usos comunicacionales más comunes de las CVM}

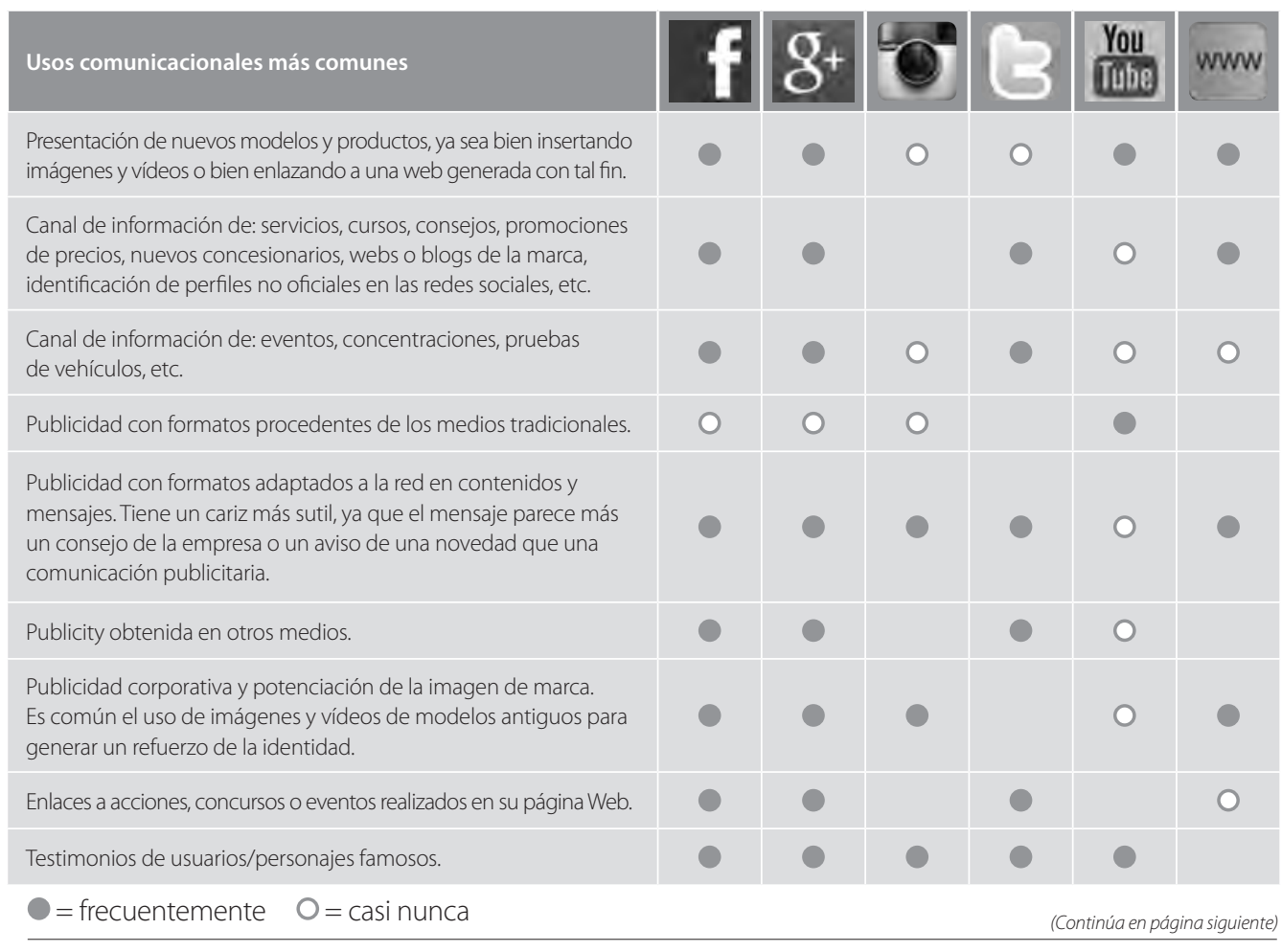




\begin{tabular}{|c|c|c|c|c|c|c|}
\hline Usos comunicacionales más comunes & & & $\mathcal{E}$ & & $\frac{\text { You }}{\text { Tohbe }}$ & wWw \\
\hline Publicidad de eventos. & 0 & 0 & & O & & $\mathrm{O}$ \\
\hline Promoción de aplicaciones para smartphones. & 0 & $\mathrm{O}$ & & ○ & & \\
\hline Publicidad de acciones conjuntas con otras marcas. & 0 & $\mathrm{O}$ & O & O & & \\
\hline Apoyo a causas sociales & 0 & ○ & & 0 & & \\
\hline$=$ frecuentemente $\quad \mathrm{O}=$ casi nunca & & & & & & \\
\hline
\end{tabular}

En general, la comunicación a través de las diferentes plataformas en las que están presentes las CVM tiene las siguientes características:

- Se potencian las sinergias entre las redes.

- No se permite que los usuarios inicien un tema, sino que esta acción es competencia exclusiva del community manager, por lo que la participación creativa de los usuarios se limita a comentar los contenidos, ya sean comerciales o no.

- El diseño de los perfiles en las distintas redes sociales son empleados generalmente para mostrar nuevos modelos o vehículos, así como con publicidad de ellos.

- Es muy común que se lancen mensajes para distintas épocas del año (p. ej.: carnaval, día del padre, Navidad, etc.), aunque sin fines comerciales directos.

- La mayoría utiliza enlaces o retwittea noticias o información que aparecen en otros medios, especialmente en el caso de las motocicletas.

- La mayoría de las historias contadas en las plataformas parten de la propia empresa.

- La información comercial se basa principalmente en la presentación y lanzamiento de nuevos modelos, mejoras, servicios ofrecidos, etc. Esto se hace de dos formas: a) mediante un mensaje acompañado de imágenes o vídeos que invitan a probar y conocer el nuevo producto; b) directamente mediante la inserción de anuncios comerciales de texto, vídeo o fotografía.

- Es muy común el empleo de personas famosas para promocionar los productos, sobre todo, deportistas y pilotos.

- En la práctica totalidad de las comunidades se informa sobre eventos, concursos, salones, ferias, encuentros, etc. que son promovidos por la empresa o en los que ésta participa.

- Todas las redes permiten a los usuarios compartir los contenidos generados por la marca. Por ello, todas las acciones buscan cierto componente de viralidad, de forma que ayude a expandir el mensaje incluyendo a no usuarios de la comunidad.

- La presencia de la marca en distintas plataformas no conlleva, por lo general, que los mensajes se adapten a ellas. En consecuencia, se reproducen los mismos formatos en redes como Facebook y Google+, mientras que se modifican levemente en Twitter ya que en esta plataforma la interacción suele ser mayor mediante el uso de concursos, preguntas, retwiteos, etc. Por otra parte, 
Instagram y Youtube se utilizan para generar contenidos para el resto de plataformas además de como repositorio de imágenes y vídeos creados por las empresas.

- Las comunidades no son empleadas actualmente, o al menos de una manera activa y frecuente, para acciones de venta personal o de marketing directo.

\section{Conclusiones}

Como se ha puesto de manifiesto las principales empresas del sector de automoción tienen una presencia notable en las redes sociales y utilizan profusamente las CVM para acercar sus mensajes a los usuarios, reproduciéndolos simultáneamente en más de una plataforma, en ocasiones aprovechando las sinérgias y en otras simplemente por complementariedad de medios o reiteración del mensaje. Las CVM se están utilizando principalmente para la comunicación de eventos, acciones y promociones, tal vez excesivamente, sin que se visualice claramente el fortalecimiento de la imagen de marca con esta política de comunicación. Además, en muchos casos los contenidos se reproducen sin adaptarlos a las peculiaridades de la red y en otros se limitan a la difusión de imágenes o vídeos.

Por otra parte, si bien la popularidad de las plataformas utilizadas facilita la penetración de las CVM entre los usuarios, el uso que de ellas están haciendo las empresas no favorece la interacción de los usuarios ni el sentido de pertenencia. Es más, en algunas comunidades se aprecia un excesivo control de los mensajes por parte del community manager.

Por lo tanto, se considera que las marcas deberían modificar el uso que están haciendo de las CVM para favorecer la comunicación adaptada al medio, motivar la participación y promover el sentido de pertenencia. Para ello la comunidad debe disponer de más información sobre el perfil de sus usuarios de manera que pudiera generar más valor atendiendo a sus características particulares (estilo de vida, intereses, ciclo de vida familiar, etc.). La CVM debe buscar la participación e interacción de los usuarios y no meramente la difusión de comunicación unidireccional que, en algunos casos, incluso se realiza en un idioma distinto del que se le presupone al usuario del país. Con tal fin no solo sería deseable el desarrollo de más acciones que propicien la interacción, sino incluso promover actividades cross channel que combinen los ámbitos online y offline.

Por último, este trabajo no está exento de limitaciones. A pesar de que se ha incluido un número importante de CVM que operan en diferentes plataformas, el método del caso no permite precisar la frecuencia de uso de cada tipo de comunicación por lo que futuros trabajos podrían abordar esta cuestión a través de un estudio de naturaleza cuantitativa longitudinal. Además, el trabajo se podría enriquecer incluyendo información procedente tanto de los usuarios como de los community managers, lo que ampliaría la perspectiva del análisis y la validez de sus resultados. 


\section{Bibliografía}

Andersen, P. H. (2005). Relationship marketing and brand involvement of professionals through web-enhanced bran communities: the case of Coloplast. Industrial Marketing Management, 34(1), 39-51.

Anesdor - Asociación Nacional de Empresas del Sector de Dos Ruedas (2014): Rankings Top15 por marca. Marzo 2014. Disponible en: https://www.anesdor.com/recursos/ estadisticas/documentos/doc_285.pdf

Apaolaza, V., Hartmann, P., Medina, E., Barrutia, J. M., y Echebarria, C. (2013). The relationship between socializing on the Spanish online networking site Tuenti and teenagers' subjective wellbeing: The roles of self-esteem and loneliness. Computers in Human Behavior, 29(4), 1282-1289.

Bagozzi, R. P. y Dholakia, U. M. (2002). International social action in virtual communities. Journal of Interactive Marketing, 16(2), 2-21.

Bagozzi, R. P. y Dholakia, U. M. (2006). Antecedents and purchase consequences of customer participation in small group brand communities. International Journal of Research Marketing, 23(1), 45-61.

Berger, S. C. y Messerschmidt, C. M. (2009). Babbling before banking. Online communities and pre-purchase information seeking. International Journal of Bank Marketing, 27(6), 446-466.

Bickart, B. y Schindler, R. M. (2001). Internet forums as influential sources of consumer information. Journal of Interactive Marketing, 15(3), 31-40.

Brown, S., Kozinets, R. V. y Sherry, J. F. Jr. (2003). Teaching old brands new tricks: Retro branding and the revival of brand meaning. Journal of Marketing, 67(3), 19-33.

Casaló, L. V., Flavián, C. y Guinalíu, M. (2007). The impact of participation in virtual brand communities on consumer trust and loyalty: the case of free software. Online Information Review, 31(6), 775-792.

Casaló, L. V., Flavián, C. y Guinalíu, M. (2010). Relationship quality, community promotion and brand loyalty in virtual communities: Evidence from free software communities. International Journal of Information Management, 30(4), 357-367.

Casaló, L. V., Flavián, C. y Guinalíu, M. (2011). New members' integration: Key factor of success in online travel communities. Journal of Business Research, 66(6), 706-710.
Cheung, C. M. K., Chiu, P-Y. y Lee, M. K. O. (2011). Online social networks: Why do students use Facebook?. Computes in Human Behavior, 27(4), 1337-1343.

Clemons, E. K. (2009). The complex problem of monetizing virtual electronic social networks. Decision Support Systems, 48(1), 46-56.

Cortés, M. y Martínez-Priego, J. M. (2010). El nuevo marketing y la figura del community manager. Revista Harvard Deusto Marketing y Ventas, 96, 16-24.

Dans, E. (2007, 9 de noviembre). La Incógnita de la publicidad en las redes sociales. Blog de investigación y opinión acerca de los sistemas y tecnologías de la información. Disponible en: http://www.enriquedans.com/2007/11/la-incognitade-la-publicidad-en-las-redes-sociales.html.

De Salas, M. I. (2009). La publicidad en las redes sociales: de lo invasivo a lo consentido. Icono 14, 15, 75-84.

Dholakia, U. M., Bagozzi, R. y Pearo, L. K. (2004). A social influence model of consumer participation in network and small group based virtual communities. International Journal of Research in Marketing, 21(3), 241-263.

Dick, A. S. y Basu, K. (1994). Customer loyalty: toward an integrated conceptual framework, Journal of the Academy of Marketing Science, 22(2), 99-113.

Etzioni, A. (1996). The responsive community: A communitarian perspective. American Sociological Review, 61(1), 1-11.

Farquhar, J. y Rowley, J. (2006). Relationships and online consumer communities. Business Process Management, 12(2), 162-177.

Félix, R. (2012). Brand communities for mainstream brands: the example of the Yamaha Rl brand community. Journal of Consumer Marketing, 29(3), 225-232.

Flavián, C. y Guinalíu, M. (2005). The influence of virtual communities on distribution strategies in the internet. International Journal of Retail \& Distribution Management, 33(6), 405-425.

Franke, N. y Shah, S. K. (2003). How communities support innovative activities: An exploration of assistance and sharing among end-users. Research Policy, 32(1), 157-178.

Hanna, R., Rohm, A. y Crittenden, V. L. (2011). We're all connected: The power of the social media ecosystem. Business Horizons, 54(3), 265-273. 
Henning-Thurau, T., Winner, K. P. y Gremmler, D. D. (2002) Relationship marketing outcomes. Journal of Service Research, 4(3), 230-247.

Henri, F. Y Pudelko, B. (2003): Understanding and analyzing activity and learning in virtual communities, Journal of Computer Assisted Learning, 19 (4), pp. 474-487.

Hoffman, D. L. y Novak, T. P. (1997). A New Marketing Paradigm for Electronic Commerce. The Information Society, 13, 43-57.

IabSpain (2014): V Estudio Anual de Redes Sociales. Disponible en: http://www.iabspain.net/wp-content/uploads/ downloads/2014/04/V-Estudio-Anual-de-Redes-Socialesversi\%C3\%B3n-reducida.pdf

Jang, H., Olfman, L., Ko, I., Koh, J. y Kim, K. (2008). The Influence of On-Line Brand Community Characteristics on Community Commitment and Brand Loyalty. International Journal of Electronic Commerce, 12(3), 57-80.

Jung, Y. y Kang, H. (2010). User goals in social virtual worlds: A means-end chain approach. Computers in Human Behavior, 26(2), 218-225.

Kalyanam, K. y Mcintyre, S. (2002). The E-Marketing Mix: A contribution of the E-Tailing Wars. Journal of the Academy of Marketing Science, 30(4), 487-499.

Kaplan, A. M. y Haenlein, M. (2010). Users of the world, unite! The challenges and opportunities of social media. Business Horizons, 53(1), 59-68.

Keating, B., Rugimbana, R. y Quazi, A. (2003). Differentiating between service quality and relationship quality in cyberspace. Managing Service Quality, 13(3), 217-232.

Koh, J. y Kim, Y. G. (2004). Knowledge sharing in virtual communities: A e-business perspective. Expert Systems with Applications, 26(2), 155-166.

Kotler, P., Kartajaya, H. y Setiawan, I. (2010). Marketing 3.0. From Products to Customers to the Human Spirit, Hoboken, Nueva Jersey (Estados Unidos): John Wiley \& Sons.

Kozinets, R. V. (2002). The field behind the screen: using netnography for marketing research in online communities. Journal of Marketing Research, 39(1), 61-72.

Kucuk, S. U. y Krishnamurthy, S. (2007). An analysis of consumer power on the internet. Technovtion, 27(1-2), 47-56.

Laroche, M., Habibi, M. R., Richard, M-O. y Sankaranarayanan, R. (2012). The effects of social media based brand communities on brand community markers, value creation practices, brand trust and brand loyalty. Computers in $\mathrm{Hu}$ man Behavior, 28(5), 1755-1767.

Lee, F. S., Vogel, D. y Moez, L. (2003). Virtual community informatics: A review and research agenda. Journal of Information Technology Theory and Application, 5(1), 47-61.

Leimeister, J. M., Sidiras, P. y Krcmar, H. (2004). Success factors of virtual communities from the perspective of members and operators: an empirical study. Proceedings of the 37th Hawaii International Conference on System Sciences, Hawaii, Estados Unidos.

Li, H. (2004). Virtual community studies: a literature review, synthesis and research agenda. Proceedings of the Americas Conference on Information Systems, Nueva York, Estados Unidos.

Lin, K-Y. y Lu, H-P. (2011). Why people use social networking sites: An empirical study integrating network externalities and motivation theory. Computers in Human Behavior, 27(3), 1152-1161.

Marta, C., Martínez, E. y Sánchez, L. (2013). La «i-Generación» y su interacción en las redes sociales. Análisis de Coca-Cola en Tuenti. Comunicar, 40, 41-48.

Martínez-López, F. J. y Luna, P. (2008). Marketing en la Sociedad del Conocimiento. Madrid: Delta Publicaciones.

Marzocchi, G., Morandin, G. y Bergami, M. (2012). Brand communities: loyal to the community or to the brand? European Journal of Marketing, 47(1), 93-114.

McAlexander, J. H., Schouten, J. W. y Koenig, H. F. (2002). Building brand community. Journal of Marketing, 66(1), 38-54. McWilliam, G. (2000). Building stronger brands through online communities. Sloan Management Review, 41(3), 43-54.

Meerman, D. (2007). The new rules of Marketing and PR, Hoboken, Nueva Jersey (Estados Unidos): John Wiley \& Sons. MSI Sistemas de Inteligencia de Mercado (2013): Termómetro de ventas de automóviles en España 2013. Documento inédito.

Muñiz, A. M. y O'Guinn, T. C. (2001). Brand Communities. Journal of Consumer Research, 27(4), 412-432.

Muñiz, A. y Schau, H. J. (2005). Religiosity in the abandoned Apple Newton brand community. Journal of Consumer Research, 31(4), 737-47.

Muntinga, D. G., Moorman, M. y Smit, E. G. (2011). Introducing COBRA's: Exploring Motivations for brand-related 
social media use. International Journal of Advertising, 30(1), $13-46$.

Porter, C. E. (2004). A Typology of Virtual Communities A Multi-Disciplinary Foundation for Future Research, Journal of Computer-Mediated Communication, 10 (1), artículo 3.

Porter, C. E. y Donthu, N. (2008). Cultivating trust and harvesting value in virtual communities. Management Science, 54(1), 113-128.

Powell, G. R., Groves, S. W. y Dimos, J. (2011). ROI of the Social Media. Hoboken, Nueva Jersey (Estados Unidos): John Wiley \& Sons.

Preece, J. (2000). Online Communities: Designing Usability, Supporting Sociability. Chichester (Reino Unido): John Wiley \& Sons.

Raney, A. A., Arpan, L. M., Pashupati, K. y Brill, D. A. (2003). At the movies, on the web: An investigation of the effects of entertaining and interactive web content on site and brand evaluations. Journal of Interactive Marketing, 17(4), 38-53.

Rheingold, H. (1993). The Virtual Community. Reding (Estados Unidos): Addison Wesley.

Sánchez-Franco, M. J. y Rondán-Cataluña, F. J. (2010). Virtual travel communities and customer loyalty: Customer purchase involvement and web site design. Electronic Commerce Research and Applications, 9(2), 171-182.

Schouten, J. W. y McAlexander, J. H. (1995). Subcultures of Consumption: An Ethnography of the New Bikers. Journal of Consumer Research, 22(1), 43-61.

Shang, R-A., Chen, Y-C. y Liao H-J. (2006). The value of participation in virtual consumer communities on brand loyalty. Internet Research, 16(4), 398-418.

Sicilia, M. y Palazón, M. (2010). El papel de las redes sociales online en la difusión de innovaciones. Libro de Actas del XXII Congreso Nacional de Marketing, AEMARK 2010.

Singh, S. y Sonnenburg, S. (2012). Brand Performances in Social Media. Journal of Interactive Marketing, 26(4), 189197.

Smedberg, A. (2008). Learning conversations for people with established bad habits: A study of four health commu- nities. International Journal of Healthcare Technology \& Management, 9(2), 143-154.

Sung, Y., Kim, Y., Kwon, O. y Moon, J. (2010). An explorative study of Korean consumer participation in virtual brand communities in social network sites. Journal of Global Marketing, 23(5), 430-445.

Wang, E. S-T. y Chen, L. S-L. (2012). Forming relationship commitments to online communities: The role of social motivations. Computers in Human Behavior, 28(2), 570-575.

Weber, L. (2007). Marketing to the Social Web. How digital customer communities build your business. Hoboken, Nueva Jersey (Estados Unidos): John Wiley \& Sons.

Wellman, B., Salaff, J., Dimitrova, D., Garton, L., Gulia, M. y Haythornthwaite, C. (1996). Computer networks as social networks: Collaborative work, telework, and virtual community. Annual Review of Sociology, 22(1), 213-238.

Wiegandt, P. (2009). Value Creation of Firm-Established Brand Communities. Wiesbaden (Alemania): Gabler.

Wirtz, J. Den Ambtman, A., Bloemer, J., Horvath, C., Ramaseshan, B. Van De Klundert, J., Gurhan, Z. y Kandampully, C. J. (2013). Managing brands and customer engagement in online brand communities. Journal of Service Management, 24(3), 223-244.

Yang, N. (2010). The Experiential Marketing in the Online Community. Web Society (SWS), IEEE 2nd Symposium, 454457.

Yeh, Y. H. y Choi, S. M. (2011). MINI-lovers, maxi-mouths: An investigation of antecedents to eWOM intention among brand community members. Journal of Marketing Communications, 17(3), 145-162.

Yen, J. R. y Gwinner, K. P. (2003). Internet retail customer loyalty: the mediating role of relational benefits. International Journal of Service Industry Management, 14(5), 483-500.

Zaglia, M. E. (2013). Brand communities embedded in social networks. Journal of Business Research, 66(2), 216-223.

Zheng, Y. M., Zhao, K. y Stylianou, A.C. (2013). The impacts of information quality and system quality on users' continuance intention in information-exchange virtual communities: An empirical investigation. Decision Support Systems, 56, 513-524. 
